\title{
A GENERALIZED DYNAMIC COMPOSITION ALGORITHM OF WEIGHTED FINITE STATE TRANSDUCERS FOR LARGE VOCABULARY SPEECH RECOGNITION
}

\author{
Octavian Cheng ${ }^{1,2}$, John Dines ${ }^{1}$ and Mathew Magimai Doss ${ }^{3}$ \\ ${ }^{1}$ IDIAP Research Institute, Martigny, Switzerland \\ ${ }^{2}$ Department of Electrical and Computer Engineering, The University of Auckland, New Zealand \\ ${ }^{3}$ International Computer Science Institute, Berkeley, California, USA \\ Email: \{ocheng, dines\}@idiap.ch, mathew@icsi.berkeley.edu
}

\begin{abstract}
We propose a generalized dynamic composition algorithm of weighted finite state transducers (WFST), which avoids the creation of noncoaccessible paths, performs weight look-ahead and does not impose any constraints to the topology of the WFSTs. Experimental results on Wall Street Journal (WSJ1) 20k-word trigram task show that at $17 \%$ WER (moderately-wide beam width), the decoding time of the proposed approach is about $48 \%$ and $65 \%$ of the other two dynamic composition approaches. In comparison with static composition, at the same level of $17 \%$ WER, we observe a reduction of about $60 \%$ in memory requirement, with an increase of about $60 \%$ in decoding time due to extra overheads for dynamic composition.
\end{abstract}

Index Terms - Weighted Finite State Transducers, Dynamic Composition, Large Vocabulary Continuous Speech Recognition

\section{INTRODUCTION}

Recently, the use of Weighted Finite State Transducers (WFST) for Large Vocabulary Continuous Speech Recognition (LVCSR) has become an attractive approach $[1,2]$. In simple terms, a WFST is a finite state machine which maps sequences of input symbols to sequences of output symbols with an associated weight. In the application of WFST in LVCSR, the idea is to represent each individual knowledge source by a WFST and fully integrate them into a unified WFST by the composition algorithm [2]. The fully integrated WFST provides weighted mappings from HMM state sequences to word sequences. Thus the speech recognition problem becomes searching for the mapped sequence with the lowest associated weight (cost). The composition of knowledge sources is a one-off process and is done offline. Therefore it is often referred to static composition.

There are two main advantages with the static approach. First the decoder design is simple because all the knowledge sources are integrated into one compact WFST. The knowledge sources are $d e$ coupled from the Viterbi search and therefore the decoder does not need to perform any combination of knowledge sources during decoding. The second advantage is that the fully integrated transducer can be further optimized by algorithms, such as, determinization, minimization and weight-pushing $[1,3]$.

Despite the above advantages, there are several drawbacks with the static approach. They include:

This work was supported by the EU 6th FWP IST integrated project AMI and the Swiss National Science Foundation through the National Center of Competence in Research (NCCR) on "Interactive Multimodal Information Management (IM2)"
- The composition and optimization of the fully integrated WFST has prohibitively high memory requirement when the constituent WFSTs are large and complex;

- The size of the fully integrated WFST can be very large, resulting in large memory requirement during decoding;

- It does not allow on-line modification of knowledge sources once they have been fully integrated.

One way of addressing these issues is to perform dynamic transducer composition during decoding. Instead of representing the entire search space by an optimized transducer, it is possible to factorize the search space into two or more transducers. These component transducers are built statically and optimized separately. The combination is done dynamically during decoding.

In this paper, we investigate several existing dynamic composition approaches and propose our improved algorithm, which avoids the creation of non-coaccessible transitions, performs weight lookahead and does not impose any constraints to the topology of component WFSTs. The paper is organized as follows. Section 2 briefly describes static WFST composition and how a fully integrated WFST is generated. Section 3 gives a general overview on current approaches to dynamic WFST composition. Section 4 describes our dynamic composition algorithm. Experimental results on different composition methods are shown in Section 5. Finally, Section 6 concludes the paper.

\section{STATIC WFST COMPOSITION}

Static WFST composition involves integration of all the knowledge sources. It can be represented by the following expression [2].

$$
N=\pi_{\epsilon}(\min (\operatorname{det}(\tilde{H} \circ \operatorname{det}(\tilde{C} \circ \operatorname{det}(\tilde{L} \circ G)))))
$$

In the above expression, $\tilde{H}$ represents the HMM topology; $\tilde{C}$ is a WFST which maps context-dependent phones to context-independent phones; $\tilde{L}$ is the lexicon WFST and $G$ is the language model (LM) WFST. The symbol $\circ$ is the composition operator. Transducer optimization algorithms, for example determinization and minimization, are represented by det and min operators respectively. The . symbol means that the WFST is augmented with auxiliary symbols which are necessary for the success of transducer optimization. The $\pi_{\epsilon}$ operation replaces the auxiliary symbols by $\epsilon$ (null) symbols. The final transducer $N$ is a fully integrated transducer which maps HMM state sequences to word sequences. 


\section{CURRENT APPROACHES TO DYNAMIC WFST COMPOSITION}

Several groups of researchers have proposed different approaches to dynamic WFST composition. They include Dolfing [4], Willett [5], Caseiro [6] and Hori [7]. The first step of any dynamic composition algorithm is to factorize the entire search space into two or more component WFSTs before decoding. Approaches include:

1. Separating the entire $G$ from other knowledge sources, resulting in two WFSTs [6];

2. Separating only part of the $G\left(G_{i}\right.$ or so called the incremental LM) from other knowledge sources. The remaining part of the $\mathrm{LM}\left(G_{s}\right.$ or the smearing $\left.\mathrm{LM}\right)$ is statically composed with other knowledge sources, resulting in two WFSTs [4, 5];

3. Factorizing the entire search space into multiple WFSTs [7].

During decoding, the component WFSTs are composed on-thefly. There are two main approaches for combining component transducers dynamically, namely with no look-ahead and with look-ahead.

The no look-ahead approach is basically the dynamic version of static WFST composition. When two WFSTs, for example $\tilde{L}$ and $G$, are composed, the $\epsilon$-output labels of $\tilde{L}$ are treated as "free-entries". They are not mapped with the input labels of $G$. These transitions are duplicated into the composite transducer, which is $\tilde{L} \circ G$ in this example.

There are two problems with this approach. The first problem is the creation of non-coaccessible transitions or so called "dead-end" transitions [6]. They are the transitions which will not reach the final state of a transducer. The second problem is the delay of the application of transducer weights. Weights in $G$ are not applied to the composite transducer until there is an actual mapping between the output symbols and the input symbols of the component transducers. For pruning efficiency, it is beneficial to introduce $G$ weights as early as possible before the actual mapping of symbols occurs, hence the motivation for the incremental approach.

The look-ahead approach proposed by Caseiro [6] addresses the above problems. He subdivides $L$ into two regions, a prefix region and a suffix region. The prefix region is the region between the initial state of $\tilde{L}$ and a non- $\epsilon$ output transition. In Figure 1, the prefix region is bounded by the grey rectangle. The region between the non- $\epsilon$ output transitions and the final state is the suffix region, which is bounded by the white rectangle. A set of anticipated output labels for each $\epsilon$-output transitions is built inside the prefix region. The function of the anticipated label sets is to provide some look-ahead information. An $\epsilon$-output transition in $\tilde{L}$ will be expanded in the composition only if there is a match between its anticipated label set and the input labels of $G$.

The early application of $G$ weights before encountering the actual non- $\epsilon$ output labels in $\tilde{L}$ can also be done by finding the semiringsum $(\oplus)$ of the weights of the matched $G$ transitions. In a tropical semiring, the $\oplus$ operator is $\min$. Thus, it is very similar to language model look-ahead [8], where partial language model weights are applied to tokens before reaching the leaf nodes (word-end nodes) of a lexical tree.

\section{PROPOSED APPROACH TO DYNAMIC WFST COMPOSITION}

We base our approach on that of Caseiro. Specifically, two component WFSTs are built: $\left(\tilde{C}_{o p t} \circ \tilde{L}_{o p t}\right)$ and $G$, where $\tilde{C}_{o p t}$ and $\tilde{L}_{o p t}$ are

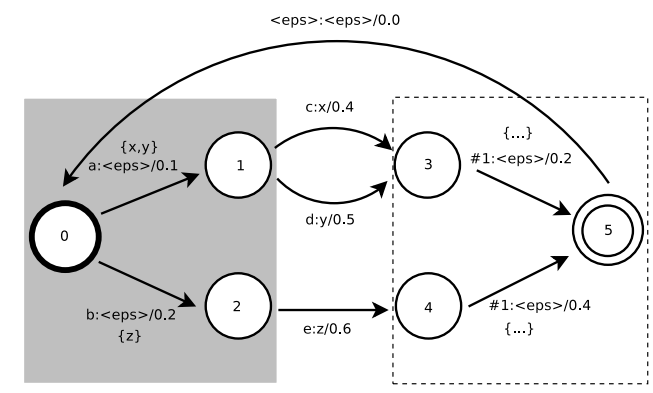

Fig. 1. The lexicon WFST $(\tilde{L})$ in Caseiro's approach. \{\} indicates an anticipated output label set. $\{\ldots\}$ means the set containing all symbols. \#1 is the word-end marker.

$\min (\operatorname{det}(\tilde{C}))$ and $\min (\operatorname{det}(\tilde{L}))$ respectively. Component transducers are combined with look-ahead, avoiding the creation of "deadend" transitions. Early application of $G$ weights is also performed.

There are however two major differences between our approach and Caseiro's approach. In [6], he presented a specialized algorithm to compose $\tilde{L}$ and $G$. He made two assumptions (or constraints) about his approach. They are:

- $\tilde{L}$ is an acyclic graph, apart from the loop which connects the final state of $\tilde{L}$ to the initial state (Figure 1)

- No weight look-ahead is performed in the suffix region.

While the first assumption holds for a typical lexicon, it is not true for an arbitrary WFST. For example, the $\left(\tilde{C}_{o p t} \circ \tilde{L}_{o p t}\right)$ WFST is cyclic in general. For the second assumption, no weight lookahead is performed in the suffix region. However, in order to achieve better pruning efficiency, weights should be distributed or "pushed" to the initial state as far as possible. Hence, look-ahead of weights, as well as the avoidance of non-coaccessible transitions, should also be performed in the suffix region.

In the following subsections, we describe how the anticipated output label sets are found in the $\left(\tilde{C}_{o p t} \circ \tilde{L}_{o p t}\right)$ transducer. We also describe how this transducer is dynamically composed with $G$ during decoding.

\subsection{Finding the Anticipated Output Labels}

The entire $\left(\tilde{C}_{\text {opt }} \circ \tilde{L}_{\text {opt }}\right)$ transducer is subdivided into prefix regions. Each prefix region is terminated with non- $\epsilon$ output label transitions. All the other transitions are $\epsilon$-output transitions.

Figure 2 illustrates an example of a cyclic $\left(\tilde{C}_{o p t} \circ \tilde{L}_{o p t}\right)$ transducer. For simplicity, only the output labels are shown. The transducer is segmented into three prefix regions. Each of them is ended with non- $\epsilon$ output label transitions. The anticipated output label set can be found by a simple depth-first traversal algorithm.

Since the transducer is subdivided into segments of prefix regions, word-end markers are now inside each region. When lookahead is carried out within each region during dynamic composition, it implies that transducer weights of $G$ could be pushed forward across the word-end markers, that is, across the word boundaries.

\subsection{The Dynamic Composition Algorithm}

The dynamic composition algorithm follows a token passing paradigm [9]. Each token holds an alignment of hypothesized words together with the corresponding accumulated cost (accCost). In dynamic 


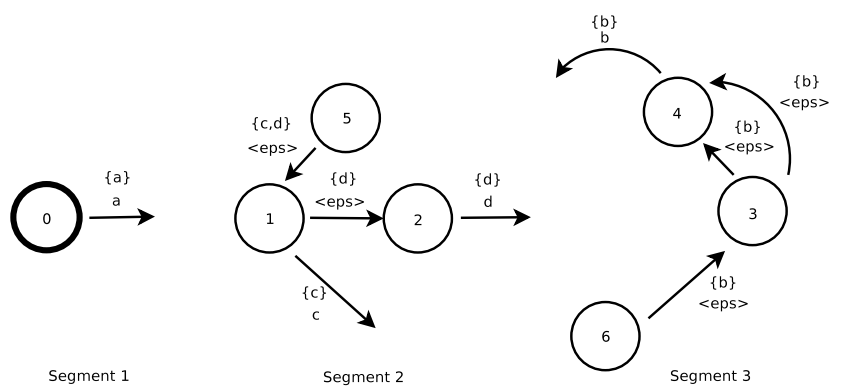

Fig. 2. A $\left(\tilde{C}_{o p t} \circ \tilde{L}_{o p t}\right)$ WFST is segmented into prefix regions, where label and weight look-ahead is performed.

composition, tokens reside in the $\left(\tilde{C}_{o p t} \circ \tilde{L}_{o p t}\right)$ transducer. Each token also has a reference to the $G$ transducer. This reference is necessary for distinguishing two tokens when they arrive at the same transition in the $\left(\tilde{C}_{o p t} \circ \tilde{L}_{o p t}\right)$ transducer, but have different word histories (i.e. different state number in the $G$ transducer). Hence, two additional attributes are required for each token. They are $S_{G}$ and pushedCost, where $S_{G}$ is the state number of the $G$ transducer to which the token is referencing and pushedCost is the accumulated look-ahead weight that has already been applied to the token.

Table 1 shows the pseudocode of the dynamic composition algorithm. The following points highlight the important parts of the algorithm.

Step 1 - 2 Update $S_{G}$ and reset pushedCost if the token is leaving a prefix region and entering a new prefix region.

Step 5a Avoid tokens entering non-coaccessible transitions.

Step 5b - 5e Perform weight look-ahead.

Step $5 f$ Organize tokens in lists. The $U D$ list allows multiple tokens with different $S_{G}$ on the same transition. The $D$ list arranges tokens according to their next_ $S_{G}$ references. This enables early recombination of tokens with the same next_S $S_{G}$ but different current $S_{G}$. It simulates suffix sharing as in WFST minimization and it is similar to [10].

\section{EXPERIMENTAL RESULTS}

The aim of this experiment is to compare the performance and the resource requirements of our dynamic composition algorithm with static composition and other dynamic composition approaches. The following list briefly describes the different approaches under test.

Static Perform decoding on the integrated $\left(\operatorname{opt}\left(\tilde{C}_{o p t} \circ \tilde{L}_{o p t} \circ G\right)\right)$.

Dynamic (Incremental, no look-ahead) Introduce unigram probabilities to build $\left(\tilde{C}_{o p t} \circ \tilde{L}_{o p t} \circ G_{u n i}\right)$. Dynamically compose this WFST with $G_{t r i-u n i}$, which is a trigram deviation from unigram, during decoding without look-ahead (i.e. no control on non-coaccessible paths and no weight look-ahead).

Dynamic (Caseiro) Build $\left(\tilde{C}_{o p t} \circ \tilde{L}_{o p t}\right)$ and $G$ WFSTs. Dynamically compose them during decoding. Since the topology of $\left(\tilde{C}_{\text {opt }} \circ \tilde{L}_{\text {opt }}\right)$ is different from $\tilde{L}$ as in his approach, there is no direct comparison. To simulate his method, the control of non-coaccessible paths and weight look-ahead is prohibited until the token reaches a word-end marker inside a prefix region. This no look-ahead region can be considered as the suffix region as in his method. Look-ahead resumes after the token has passed the word-end marker.
1. A token resides on Transition $(i: o / w)$ between States $q 1$ and $q 2$ in $\left(\tilde{C}_{\text {opt }} \circ \tilde{L}_{o p t}\right)$. If $o$ is non- $\epsilon$, the token reaches the end of a prefix region. Go to Step 2. Otherwise, the token is still within the prefix region. Go to Step 3.

2. Set $S_{G}=n e x t_{-} S_{G}$ (See Step 5 f for details about next_S $\left.S_{G}\right)$. Reset pushedCost $=0.0$.

3. Retrieve $S_{G}$ from the token.

4. Get the set of transitions leaving from State $q 2$.

5. For each transition $t$,

(a) Get the anticipated label set of $t$. Also get a set of input labels from all the transitions leaving from State $S_{G}$ of $G$. Find the intersection between these two sets. If there is no intersection, it means that $t$ is a noncoaccessible transition, the token will not enter $t$. Go back to Step 5 for the next $t$. Otherwise, go to Step 5 b.

(b) Go through all the matched transitions at State $S_{G}$. Accumulate the semiring-sum $(\oplus)$ of the weights of all the matched transitions. This is the look-ahead weight.

(c) $\Delta$ cost $=(\text { pushedCost })^{-1} \otimes($ look-ahead weight $)$.

(d) Update $a c c C o s t=($ accCost $) \otimes(\Delta$ cost $)$.

(e) Update pushedCost $=$ look-ahead weight.

(f) Check the number of matched labels in the intersection.

- $>1$ match, put the token (indexed by $S_{G}$ ) in the $U D$ (UnDecided) list of $t$. If there is a token with the same key, keep the lower accCost token.

- Only 1 match, the next $S_{G}\left(n e x t \_S_{G}\right)$ is the destination state of the matched $G$ transition. Put the token in the $D$ (Decided) list of $t$. The token is indexed by the pair $\left(\right.$ next $_{-} S_{G}$, matched $\left._{O}\right)$ where matched $_{O}$ is the matched symbol in the intersection. Keep the token with the lower accCost if there is a token with the same key.

Table 1: Pseudocode of the proposed algorithm. States $q 1$ and $q 2$ are any arbitrary states in $\left(\tilde{C}_{\text {opt }} \circ \tilde{L}_{\text {opt }}\right)$. The symbols $\oplus$ and $\otimes$ are semiring-add and semiring-multiply respectively.

Dynamic (Our approach) Build $\left(\tilde{C}_{o p t} \circ \tilde{L}_{o p t}\right)$ and $G$ WFSTs. Dynamically compose them as described in Section 4.

The performance of different approaches was assessed using the Wall Street Journal (WSJ1) corpus [11]. Cross-word triphone HMM models were trained on the "si_tr_s" set of 38275 utterances using 39-dimensional PLPs. A trigram LM, with 19979 unigrams, 3484372 bigrams and 2949590 trigrams, was used to test the $20 \mathrm{k}$ development test set "si_dt_20" from WSJ1 database, consisting of 503 utterances. The experiment was carried out using Juicer [12, 13], which is a WFST-based LVCSR decoder developed here at IDIAP.

Figure 3 shows the word error rate (WER) against the real-time factor (RTF) of different approaches. Amongst all dynamic composition approaches, the proposed method shows better WER versus RTF characteristics. One important observation is that the proposed method significantly outperforms the other two dynamic approaches at narrow and moderately-wide beam widths. At the level of $17 \%$ WER (moderately-wide beam), the RTF of our approach is about $65 \%$ and $48 \%$ of the no look-ahead approach and Caseiro's method 


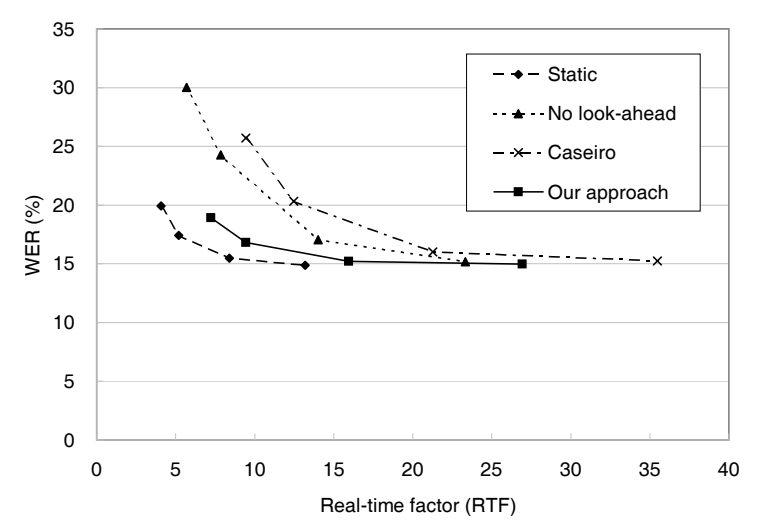

Fig. 3. WER versus RTF of different approaches at various pruning beam-widths (150, 160, 180 and 200).

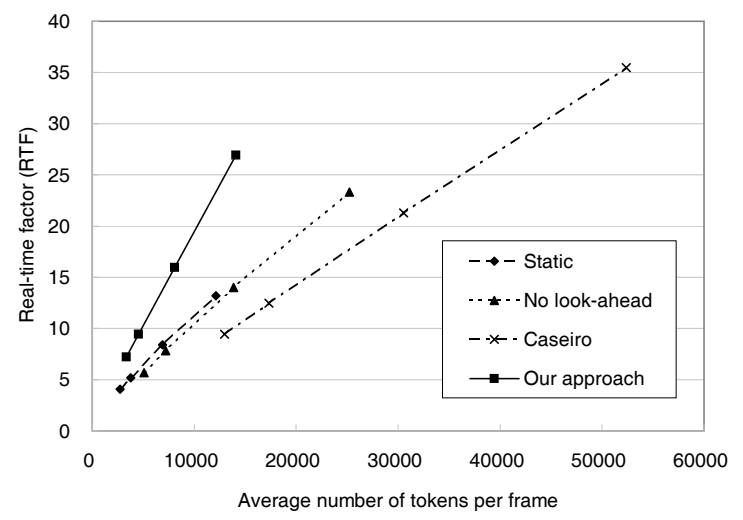

Fig. 4. RTF versus Average number of tokens per frame of different approaches at various pruning beam-widths (150, 160, 180 and 200).

respectively. This confirms that look-ahead is necessary for good accuracy-time tradeoff in narrow and moderately-wide beam width scenarios.

Comparing our approach with static composition, the WERs are similar at the same pruning settings, which suggests that our approach is close to the WFST optimization performed during static composition. At the same level of $17 \%$ WER, the RTF of the proposed approach is about $60 \%$ more than the RTF of static composition. This is due to the overhead, for example, finding the set intersection, searching tokens in a list, etc, required during dynamic composition. Figure 4 illustrates the RTF against the average number of tokens per frame. Our approach has a steeper slope in the figure, which indicates that it requires more time to process each token than the static case. Also it can be seen that the other two dynamic approaches have a lot more tokens per frame than both our approach and the static approach, which shows that the avoidance of non-coaccessible transitions in our approach helps to reduce the number of redundant tokens.

One of the major reasons to perform dynamic composition is the reduction in memory requirement. Table 2 compares the maximum memory usage (in MB) of our approach and the static approach. It shows a reduction of about $60 \%$ in memory usage.

\begin{tabular}{|l|c|c|c|}
\hline Beam width & Static & Dynamic (Our approach) & \% reduction \\
\hline 150 & 1774 & 679 & 61.7 \\
\hline 160 & 1775 & 697 & 60.7 \\
\hline 180 & 1965 & 722 & 63.3 \\
\hline 200 & 1966 & 762 & 61.2 \\
\hline
\end{tabular}

Table 2: Maximum memory usage (in MB) during decoding

\section{CONCLUSIONS}

We have proposed a generalized dynamic WFST composition algorithm, which avoids the creation of non-coaccessible transitions, performs weight look-ahead and does not impose any constraints to the topology of the WFSTs. Experimental results show that our weight look-ahead approach gives better WER versus RTF characteristics than other dynamic composition approaches. Comparing with static composition, it shows a significant reduction in memory usage.

\section{REFERENCES}

[1] M. Mohri, "Finite-state transducers in language and speech processing," Computational Linguistics, vol. 23, no. 2, pp. 269-311, 1997.

[2] M. Mohri, F. Pereira, and M. Riley, "Weighted finite state transducers in speech recognition," in ISCA ITRW Automatic Speech Recognition: Challenges for the Millennium, 2000.

[3] M. Mohri and M. Riley, "A weight pushing algorithm for large vocabulary speech recognition," in Proc. Eurospeech, 2001.

[4] H. Dolfing and I. Hetherington, "Incremental langauge models for speech recognition using finite-state transducers," in Proc. ASRU, 2001.

[5] D. Willett and S. Katagiri, "Recent advances in efficient decoding combining on-line transducer composition and smoothed language model incorporation," in Proc. ICASSP, 2002.

[6] D. Caseiro and I. Trancoso, "A specialized on-the-fly algorithm for lexicon and language model composition," IEEE Tran. on Audio, Speech and Language Processing, vol. 14, no. 4, 2006.

[7] T. Hori and A. Nakamura, "Generalized fast on-the-fly composition algorithm for WFST-based speech recognition," in Proc. Interspeech, 2005.

[8] S. Ortmanns, H. Ney, and A. Eiden, "Language-model lookahead for large vocabulary speech recognition," in Proc. ICSLP, 1996.

[9] S. Young, N. Russell, and J. Thornton, "Token passing: A simple conceptual model for connected speech recognition systems," Tech. Rep. CUED/F-INFENG/TR38, Cambridge University Engineering Department, July 1989.

[10] D. Caseiro and I. Trancoso, "A tail-sharing WFST composition algorithm for large vocabulary speech recognition," in Proc. ICASSP, 2003.

[11] D. Paul and J. Baker, "The design for the Wall Street Journalbased CSR corpus," in Proc. ICSLP, 1992.

[12] D. Moore, "The Juicer LVCSR Decoder - user manual for Juicer version 0.5.0," IDIAP-COM 03, IDIAP, 2006.

[13] D. Moore, J. Dines, M. Magimai Doss, J. Vepa, O. Cheng, and T. Hain, "Juicer: A weighted finite-state transducer speech decoder," in MLMI'06, 2006. 